\title{
Estudo econométrico dos efeitos da disponibilidade de crédito na demanda por transporte aéreo no Brasil
}

\author{
Econometric study of the effects of credit availability on air transport \\ demand in Brazil
}

\author{
Maria Catarina Torres de Carvalho ${ }^{1}$, Bruno F. Oliveira' ${ }^{2}$, Alessandro V. M. Oliveira ${ }^{3}$ \\ ${ }^{1}$ Instituto Tecnológico de Aeronáutica, Brasil, catarinacarvalho.ita@gmail.com \\ 2Instituto Tecnológico de Aeronáutica, Brasil, bfelipeoliveira@outlook.com \\ 3Instituto Tecnológico de Aeronáutica, Brasil, alessandro@ita.br
}

\section{Recebido:}

30 de novembro de 2018

Aceito para publicação:

13 de maio de 2020

Publicado:

15 de dezembro de 2020

Editor de área:

Alexandre de Barros

\section{Palavras-chaves:}

Demanda.

Consumo.

Transporte aéreo.

Econometria.

\section{Keywords:}

Demand.

Consumption.

Air transportation.

Econometrics.

DOI:10.14295/transportes.v28i5.1898

\begin{abstract}
RESUMO
A maior disponibilidade de crédito tem o potencial efeito de fomentar o consumo em uma economia, pelo menos a curto e médio prazos. No caso do transporte aéreo, pode estimular os parcelamentos de passagens aéreas, atraindo os gastos de extratos de consumidores de renda mais baixa, além de alavancar um número maior de viagens por parte do público tradicional. Adicionalmente, as empresas e corporações nacionais, com capital de giro potencialmente maior, em teoria podem dispender mais em viagens corporativas. $O$ presente trabalho desenvolve uma modelagem empírica para testar $0 \mathrm{im}$ pacto na demanda por transporte aéreo da maior concessão de crédito na economia brasileira do final dos anos 2000. Utilizando dados estatísticos obtidos junto à Agência Nacional de Aviação Civil, montou-se um painel de dados com pares de cidades brasileiras para estimar um modelo econométrico de demanda por viagens aéreas, utilizando um estimador de variáveis instrumentais. Com esse modelo, testou-se diretamente a hipótese de efeito da disponibilidade de crédito na economia local como um dos fatores explicativos da demanda da aviação comercial brasileira no período.
\end{abstract}

\begin{abstract}
Greater availability of credit has the potential effect of boosting consumption in an economy, at least in the short and medium terms. In the case of air transportation, it may stimulate the payment for airline tickets in installments, thus attracting new, lower-income consumers and leveraging a larger number of trips by the existing ones. Additionally, national companies and corporations - potentially favored with higher working capital - may, in theory, spend more on corporate travel. This paper develops an empirical model to test the impact on air transport demand of the higher amount of available credit in the Brazilian economy in the late 2000s. Using statistical data obtained from the National Civil Aviation Agency, we utilized a panel data set of Brazilian city-pairs to estimate an econometric model of air travel demand employing an instrumental variables estimator. With this approach, we were able to test the hypothesis of credit availability in the local economy as one of the explanatory factors of the Brazilian commercial aviation demand in the period.
\end{abstract}

\section{INTRODUCÃO}

A segunda metade dos anos 2000 até o início da presente década marcou um período de crescimento econômico no país, não obstante a crise financeira global de 2009. Um dos setores que se beneficiou desse maior crescimento foi o transporte aéreo que, em regime de livre 
concorrência entre as companhias aéreas, apresentou a dinâmica necessária para promover um verdadeiro salto qualitativo, apresentando taxas de crescimento sustentáveis por vários anos no período. Muito se discute, sobretudo por ocasião da posterior recessão econômica, que parte daquele boom de crescimento teria sido advindo da rápida expansão do crédito bancário, movimento este que vinha sendo observado há alguns anos na economia brasileira (Borça Jr. \& Guimarães, 2015; Galeano \& Feijó, 2012; Sant'Anna, Borça Jr. \& Araujo, 2009). De fato, pode-se levantar a hipótese de que a maior disponibilidade de crédito potencialmente fomentaria as transações econômicas reais, gerando assim a possibilidade de induzir a materialização de planos de investimento das empresas e, consequentemente, gerar maior produção e consumo. Dessa forma, o presente trabalho visa entender papel da expansão do crédito na procura por viagens aéreas no Brasil.

Na literatura, os estudos em geral apontam evidências para um efeito do crédito na economia na forma de impulso ao consumo das famílias, pelo menos a curto prazo (Olney, 1999; Di Maggio e Kermani, 2017). Sugere-se, entretanto, que a concessão de crédito pode gerar uma bolha de consumo, em um típico problema de expansão seguido de quebra econômica (boom and bust). Aponta-se também que as condições socioeconômicas dos consumidores podem ser determinantes na sua maior ou menor propensão a consumir em situação de maior disponibilidade de crédito na economia (Soman e Cheema, 2002).

0 presente trabalho promove o desenvolvimento de uma modelagem empírica a fim de analisar o impacto da maior concessão de crédito na demanda por transporte aéreo. Focando no período pré-crise econômica atual, compreendido entre 2002 e 2013, estudou-se o mercado de transporte aéreo regular doméstico. Com o aumento da disponibilidade de crédito na economia, teve-se como efeito direto a possibilidade de maior parcelamento de passagens aéreas, atraindo os gastos de extratos de consumidores de renda mais baixa, além de alavancar um número maior de viagens por parte do público tradicional. Adicionalmente, as empresas e corporações nacionais, com capital de giro potencialmente maior, em teoria poderiam ter dispendido em mais viagens corporativas. Utilizando dados estatísticos obtidos junto à Agência Nacional de Aviação Civil (ANAC), foi possível montar um painel de dados com pares de cidades brasileiras, tendo sido estimado um modelo econométrico de demanda por viagens. Com esse modelo, testou-se diretamente a hipótese de efeito da disponibilidade de crédito como um dos fatores determinantes da demanda da aviação comercial brasileira no período.

0 presente trabalho está dividido da seguinte maneira: na Seção 2 é apresentada uma análise da problemática dos modelos econométricos de demanda e a evolução das viagens aéreas no país; na Seção 3, apresenta-se a literatura dos efeitos do crédito sobre o consumo; na Seção 4 é desenvolvida a metodologia e a formulação da proposta de modelagem empírica; a Seção 5 contém os resultados das estimações; e, por fim, a Seção 6 apresenta as considerações finais.

\section{MODELOS ECONOMÉTRICOS E A EVOLUÇÃO DA DEMANDA POR VIAGENS AÉREAS NO BRASIL}

Tradicionalmente, modelos econométricos de demanda por transporte aéreo tomam por base variáveis de renda do consumidor e de preço das passagens aéreas (Kazda e Caves, 2015). Alguns exemplos na literatura incluem, por exemplo, Calderón (1997), Grosche, Rothlauf e Heinzl (2007), Valdes (2015) e Boonekamp, Zuidberg e Burghouwt (2018). Esses estudos necessariamente utilizam proxies para aquelas variáveis, renda e preço, dado que em geral são consideradas variáveis "causais", ou seja, esperadas ex-ante como fatores explicativos na relação de 
demanda. Tipicamente é também utilizado um conjunto adicional de regressores, como distância, população, tamanho da zona de influência dos aeroportos, presença de empresas de baixo custo (low costs carriers, ou LCC), dentre outros. Como variável indicadora de renda, costumase utilizar o Produto Interno Bruto (PIB), seja no seu total, seja per capita. Tendo em vista o fato do PIB sintetizar o resultado final das atividades produtivas em um país, as correlações positivas entre essa grandeza e a demanda por passagens aéreas são esperadas, podendo-se inferir que quanto maior for a produção de riqueza, maior será a demanda per capita por bens de consumo em geral - inclusive pelo transporte aéreo.

Analisando, porém, a evolução da variação real do PIB brasileiro antes da presente crise econômica, pode-se tecer considerações interessantes. Ao contrastar a variação do PIB com a variação da quantidade de passageiros pagos transportados no mercado doméstico e internacional a partir dos anos 2000, conforme Figura 1, nota-se que em 2009 o número de passageiros voados (PAX) evoluiu 14\% ao ano, ainda que o PIB brasileiro permanecesse estagnado por conta da crise financeira global. Nos anos posteriores, pode-se perceber na figura que as taxas de crescimento do transporte aéreo são consistentemente maiores que as do PIB e, mesmo no período imediatamente anterior à atual crise, quando a atividade econômica já apresentava sinais de arrefecimento, a demanda por transporte aéreo se manteve crescente. Pode-se, assim, argumentar que outros fatores devam ser considerados ao se estudar a formação da demanda por transporte aéreo no Brasil.

Ainda em relação às viagens aéreas, a segunda metade dos anos 2000 foi marcada por um boom de demanda na aviação civil (ANAC, 2016), em que ficou evidente a popularização do transporte aéreo no Brasil. As empresas aéreas parcelavam bilhetes em até trinta e seis vezes, empresas de turismo financiavam passagens no carnê sendo que, em paralelo, as operações de concessão de crédito evoluíram consideravelmente na economia brasileira. De fato, como é exibido na Figura 2, as operações de crédito ao setor privado no país apresentaram notáveis taxas de crescimento entre $11 \%$ e $25 \%$ ao ano entre 2005 e 2012 . Surge, portanto, a hipótese de que o maior acesso ao crédito e a possibilidade de parcelar passagens aéreas e sejam também fatores responsáveis pelo aumento da demanda por transporte aéreo no Brasil daquele período.

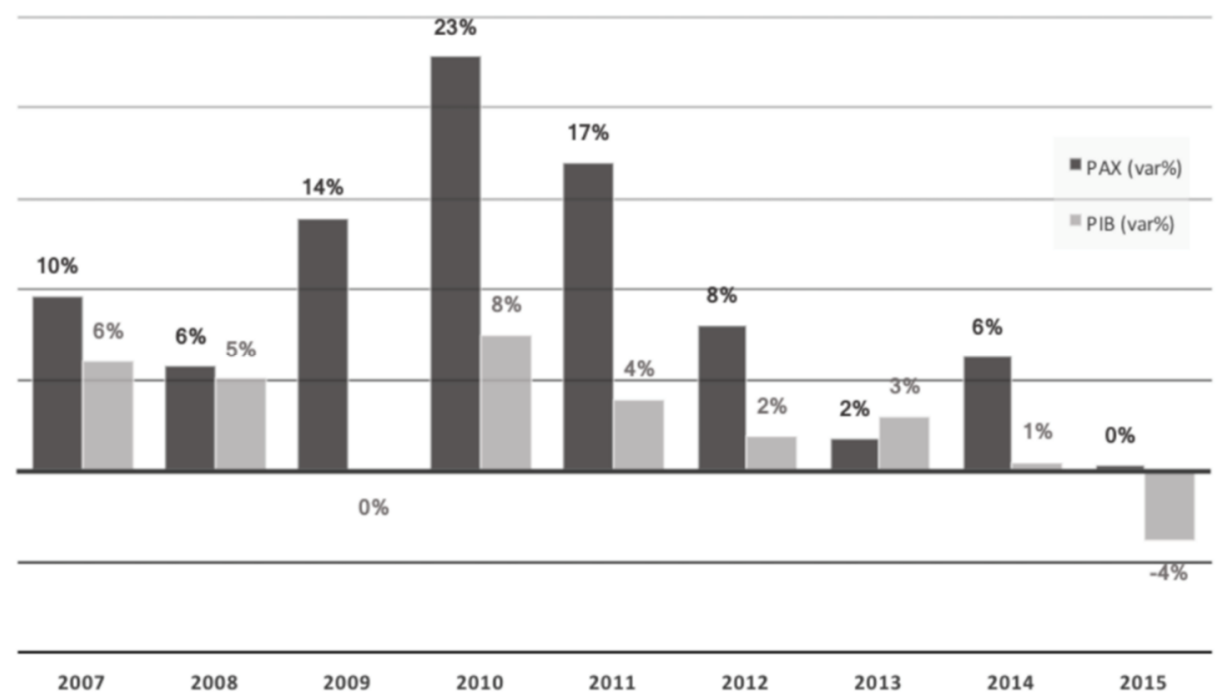

Figura 1. Variações anuais (\% ano sobre ano anterior) do crescimento número de passageiros (PAX) e do PIB. Fonte: ANAC e Ipeadata com cálculos próprios, 2002-2013 
Avaliar a potencial relação entre a disponibilidade de crédito e a demanda por passagens aéreas possibilita entender se os efeitos do incentivo ao crédito são benéficos ao setor de transporte aéreo, pelo menos no curto e médio prazos. A longo prazo, pode ser que a possibilidade de experimentação do serviço de transporte aéreo por parte de um público que antes estava à margem do consumo nesse setor, aumente a propensão ao seu consumo no futuro. Por outro lado, entretanto, o maior endividamento das famílias pode causar restrições a essa possível dinâmica intertemporal. Assim, caso as implicações dessa política sejam mais relacionadas ao endividamento dos consumidores, há a possibilidade de as empresas aéreas terem expandido suas malhas baseadas em uma projeção de demanda superestimada. Por outro lado, se o crédito atuar como um indutor de demanda e, assim, os consumidores passarem a preferir o transporte aéreo em detrimento de outros meios de locomoção, é esperado que o número de passageiros aumente não apenas enquanto perdurar o incentivo ao crédito e, portanto, a maior política de incentivo ao crédito teria sido salutar para o setor no longo prazo.

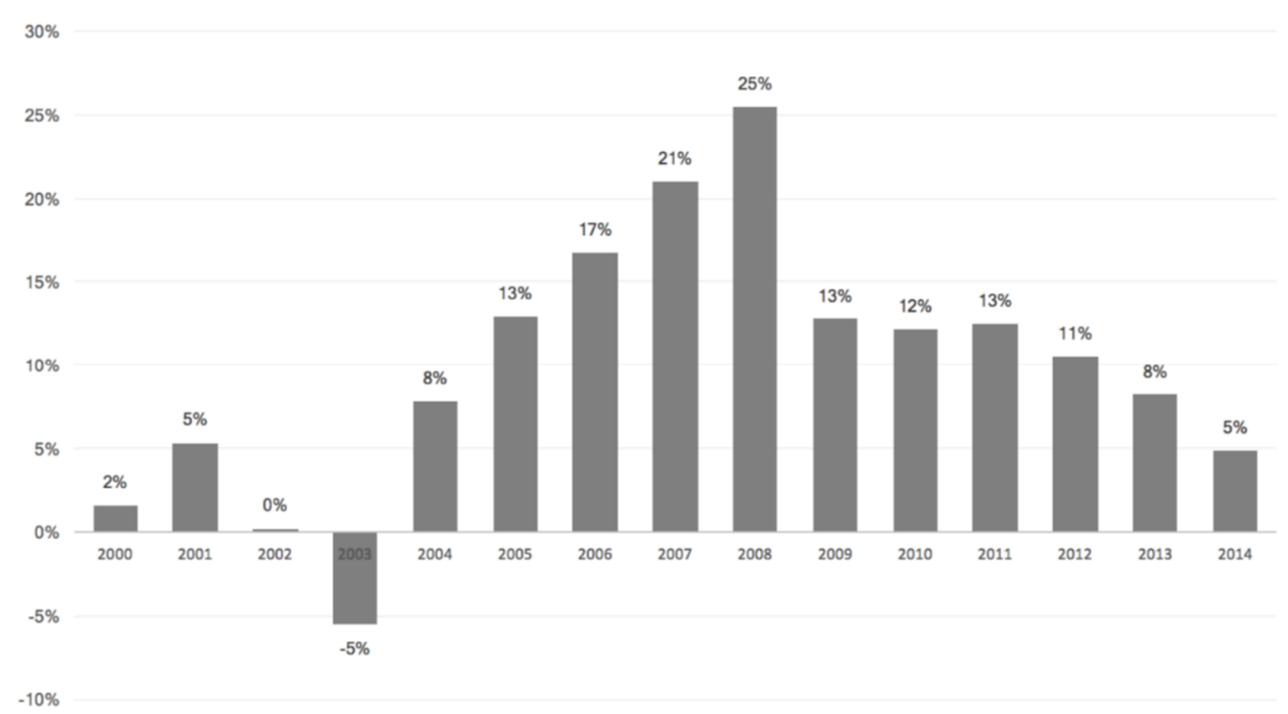

Figura 2. Variações anuais (\% ano sobre ano anterior) das operações de crédito ao setor privado. Fonte: Banco Central do Brasil, com cálculos próprios. 2002-2013

\section{A LITERATURA DOS EFEITOS DO CRÉDITO NO COMPORTAMENTO DO CONSUMIDOR}

A literatura acerca dos efeitos da disponibilidade de crédito na economia é vasta e condicional à vertente teórica macroeconômica seguida. Entretanto, em termos microeconômicos, existe uma lacuna existente na literatura sobre os efeitos da concessão de crédito em mercados de transportes de média e longa distância em geral, e, em particular do transporte aéreo. A seguir, tecemos algumas considerações a respeito da literatura econômica geral sobre o tema, como forma de promover insights para uma análise de demanda setorial.

Soman e Cheema (2002) apontam que existe um efeito relevante do crédito nas intenções de consumo em uma economia. Os autores obtêm evidências de que os efeitos da disponibilidade de crédito não apenas exercem um impacto relevante na propensão do consumidor a utilizar esse crédito para gastos em bens de consumo como, nos casos em que o consumidor é mais jovem ou possui menor grau de instrução, esse efeito é ainda mais pronunciado.

Por outro lado, temos as evidências de Di Maggio e Kermani (2017), que analisam a rápida expansão econômica seguida de crise no mercado imobiliário dos Estados Unidos dos anos 
2000. Os autores concluem que um aumento na disponibilidade de crédito possui dois principais efeitos nos preços dos imóveis, a saber: (i) no primeiro momento, um efeito de aumento nesses preços e, (ii) em seguida, uma queda brusca nos anos subsequentes. Ou seja, há evidências de que a concessão de crédito tem um potencial papel de fomentar a demanda por imóveis no curto prazo (o que aumenta os preços de mercado), gerando um problema de expansão e quebra (boom and bust). De fato, quando são analisadas as grandes crises financeiras globais (o Pânico de 1873, a Grande Depressão de 1920, a Crise Japonesa de 1991, a Crise Asiática de 1997 e a Crise dos Subprimes nos Estados Unidos em 2008), pode-se perceber que os períodos de recessão são precedidos de longos períodos de expansão de crédito (Gall, 2008). Considerando o mercado imobiliário norte-americano, Di Maggio e Kermani (2017) estabelecem paralelos entre a disponibilidade de crédito e os preços das casas nos períodos anteriores e posteriores à crise de 2008, mostrando evidências de que o crédito disponibilizado por bancos nacionais durante a recuperação econômica está intimamente relacionado ao aumento e ao subsequente colapso dos preços das casas e das taxas de empregabilidade americanas.

Na mesma linha que Di Maggio e Kermani (2017), Olney (1999), investiga um grande evento de crise, no caso a Grande Depressão dos anos 1930. A autora observa a ascensão do financiamento parcelado nos anos 1920, o que representou um marco na história do crédito ao consumidor nos Estados Unidos. Observa ainda que as compra em prestações foram responsáveis por grande parte da expansão dos anos 1920 no uso de crédito doméstico naquele país. A autora conclui que alto endividamento dos consumidores em uma economia constitui uma ameaça aos gastos futuros com consumo, especialmente em situações em que os custos com o calote (default) são muito altos.

\section{METODOLOGIA}

Na presente seção, apresentamos a proposta metodológica de abordagem do problema de investigação dos efeitos do crédito na economia sobre a demanda por transporte aéreo no Brasil durante o período imediatamente posterior à crise financeira global de 2008 e anterior ao período recessivo de meados da década de 2010. Apresentaremos, assim, a amostra de dados (4.1), o modelo econométrico proposto (4.2), a estratégia de estimação (4.3) e o procedimento de validação cruzada (4.4).

\subsection{Dados}

Os dados utilizados na pesquisa compõem um painel com informações mensais acerca da demanda por transporte aéreo em 369 rotas em operação no Brasil no período entre janeiro de 2002 e dezembro de 2013. No presente estudo, uma rota é definida como um par de cidades entre as quais há, no mínimo, um voo comercial doméstico direto. Nas capitais Rio de Janeiro, São Paulo e Belo Horizonte utiliza-se o conceito de região metropolitana estendida da cidade em questão, ou seja, dados referentes a cidades no entorno da microrregião da capital são incluídos. Os dados relativos às operações nos aeroportos estão disponíveis na Base de Dados Estatísticos do Transporte Aéreo e dados do Relatório de Tarifas Aéreas Domésticas da Agência Nacional de Aviação Civil (ANAC).

A base também engloba informações oriundas do Instituto Brasileiro de Geografia e Estatística - IBGE (em seu portal SIDRA), Instituto de Pesquisa Econômica Aplicada - IPEA (em seu portal IPEADATA), e do Banco Central do Brasil (em seu portal ESTBAN - Estatísticas Bancárias por Município). Utiliza-se o IPCA/IBGE como índice para ajuste a valores constantes das séries 
monetárias. É importante salientar que as informações acerca do PIB e da população municipal (disponibilizadas pelo IBGE e IPEA) foram manuseadas por meio de interpolação geométrica para se obter as respectivas estimativas mensais. Adicionalmente, no cômputo dessas variáveis do modelo, para fins de cômputo de valores associados ao par de cidades, considera-se um conceito de "cidade estendida", englobando a mesorregião de origem e destino para incorporar a zona de influência do(s) aeroporto(s) das cidades envolvidas. No caso da cidade de São Paulo, são também incluídas as mesorregiões de Campinas, Macro Metropolitana Paulista e Vale do Paraíba, além da Metropolitana de São Paulo.

\subsection{Modelo Econométrico}

A Equação 1 representa o modelo econométrico de demanda por transporte aéreo:

$$
\ln y_{k t}=\sum_{i=1}^{N} \beta_{i} \ln X_{i, k t}+\sum_{j=1}^{M} \delta_{j} D_{j, k t}+\gamma_{k}+u_{k t}
$$

onde ln $y_{k t}$ representa o movimento de passageiros, expresso em logaritmo neperiano, sendo observado nas dimensões $k$ (par de cidades) e $t$ (tempo, com periodicidade mensal); $\ln X_{i, k t}$ retrata a $i$-ésima variável explicativa contínua de demanda $(i=1, \ldots, I)$, também expressa em logaritmo neperiano; $D_{j, k t}$ representa a $j$-ésima variável binária ("dummy") deslocadora de demanda $(j=1, \ldots, J) ; \gamma_{k}$ representam os efeitos fixos de par de cidades e, portanto, controlam as idiossincrasias associadas aos diferentes mercados e que são constantes ao longo do tempo (distância, por exemplo); $\beta_{i}$ e $\delta_{j}$ representam os coeficientes a serem estimados; $u_{k t}$ representa o termo de erro aleatório. A Tabela 1 apresenta a descrição das variáveis utilizadas na especificação empírica do modelo. Por simplificação da exposição, no que se segue omitiremos os índices $k$ e $t$.

As estatísticas descritivas das variáveis utilizadas no modelo podem ser analisadas na Tabela 2, em que é possível observar correlações positivas das variáveis PIB e densidade populacional (POP) com o número de passageiros diários (PAX) e, interessantemente, a correlação relativamente alta entre as variáveis referentes à disponibilidade de crédito (CRD, EMP e FIN) e PAX.

\subsection{Estratégia de Estimação}

Os modelos de regressão desenvolvidos no presente trabalho foram rodados utilizando-se o chamado Estimador de Efeitos Fixos (Fixed Effects, FE). No estimador FE são extraídas as médias das variáveis para cada um dos indivíduos do painel e, na sequência, é rodada uma regressão das variáveis modificadas pela subtração dessas médias - variáveis time-demeaned, tanto o regressando quanto os regressores. 0 estimador de efeitos fixos é equivalente ao procedimento de rodar a regressão original utilizando uma variável dummy para cada indivíduo do painel (o chamado Least-Squares Dummy Variables, LSDV, vide Maddala \& Lahiri, 1992). 0 uso de efeitos fixos, nesse modelo, visa controlar as idiossincrasias invariantes no tempo de cada rota, o que significa proporcionar uma maior confiabilidade dos resultados pelo controle de possíveis fatores omitidos e que são localmente presentes (mas não observados pelo analista) nas rotas.

Uma forma de avaliar a qualidade do controle dos efeitos fixos estimados é efetuar o teste conjunto dos coeficientes das variáveis dummies no modelo LSDV. Outra forma de avaliar o desempenho do estimador de efeitos fixos é rodar testes de especificação de Hausman, comparando os seus resultados com os resultados de um estimador de efeitos aleatórios (Random Effects, RE), e mesmo com os resultados do estimador de Mínimos Quadrados Ordinário (MQ0). Em caso de diferença sistemática entre os coeficientes, opta-se por preferir o estimador de efeitos fixos por suas propriedades de melhor controle de não observáveis e, portanto, ausência 
de viés de variáveis omitidas (Wooldridge, 2016). Todos os resultados desses testes de hipóteses são apresentados na Tabela 3. Em todas as análises, confirma-se a qualidade da estimação adotada no trabalho, por meio da rejeição da hipótese nula dos respectivos testes.

Tabela 1 - Descrição das variáveis do modelo empírico

\begin{tabular}{|c|c|c|c|c|}
\hline Variável & Descritivo & $\begin{array}{l}\text { Unidade de } \\
\text { medida }\end{array}$ & Cálculo & Fonte \\
\hline PAX & $\begin{array}{l}\text { passageiros } \\
\text { voados }\end{array}$ & $\begin{array}{l}\text { Número de } \\
\text { passageiros }\end{array}$ & $\begin{array}{c}\text { Total de passageiros pagantes diários médios voados } \\
\text { (regressando). }\end{array}$ & ANAC \\
\hline PPC & PIB per capita & $\begin{array}{l}\text { R\$ constantes } \\
\text { de jan } / 2015\end{array}$ & $\begin{array}{l}\text { Média geométrica dos PIBs per capita das cidades de origem e de } \\
\text { destino. }\end{array}$ & $\begin{array}{l}\text { IPEADATA, } \\
\text { IBGE }\end{array}$ \\
\hline POP & $\begin{array}{l}\text { densidade } \\
\text { populacional }\end{array}$ & $\begin{array}{l}\text { Número de } \\
\text { residentes por } \\
\mathrm{km}^{2}\end{array}$ & $\begin{array}{c}\text { Média geométrica das densidades populacionais das cidades de } \\
\text { origem e de destino. }\end{array}$ & IBGE \\
\hline YLD & $\begin{array}{l}\text { preço por } \\
\text { quilômetro }\end{array}$ & $\begin{array}{l}\mathrm{R} \$ \text { constantes } \\
\text { de jan } / 2015\end{array}$ & $\begin{array}{l}\text { Preço médio por quilômetro. A distância geodésica média entre } \\
\text { os pares de cidades foi calculada usando a fórmula de Vincenty. }\end{array}$ & ANAC, IBGE \\
\hline LCC & $\begin{array}{l}\text { presença de } \\
\text { aérea } \\
\text { "low cost" }\end{array}$ & Binária $(0,1)$ & $\begin{array}{l}\text { dummy que considera a presença ou não das empresas Gol e } \\
\text { Azul, empresas que iniciaram suas operações no Brasil como } \\
\text { "low cost". }\end{array}$ & ANAC \\
\hline ENT & $\begin{array}{l}\text { presença de } \\
\text { demais aéreas } \\
\text { entrantes }\end{array}$ & Binária $(0,1)$ & $\begin{array}{c}\text { dummy que considera a presença ou não das empresas Webjet, } \\
\text { BRA e Avianca; essas empresas possuíam operação de menor } \\
\text { porte e entraram no mercado doméstico em períodos posteriores } \\
\text { ao início da amostra, sendo a variável indicativa do grau de } \\
\text { contestabilidade dos mercados (facilidade ou dificuldade de } \\
\text { entrada de rivais menores). }\end{array}$ & ANAC \\
\hline $\mathrm{CSH}$ & $\begin{array}{l}\text { acordo } \\
\text { codeshare }\end{array}$ & Binária $(0,1)$ & $\begin{array}{c}\text { dummy que controla o período e rotas do acordo de } \\
\text { compartilhamento de aeronaves entre as companhias Varig e } \\
\text { TAM, entre } 2003 \text { e } 2005 .\end{array}$ & $\begin{array}{l}\text { MIN. FA- } \\
\text { ZENDA }\end{array}$ \\
\hline CRD & crédito & $\begin{array}{l}\text { R\$ constantes } \\
\text { de jan } / 2015\end{array}$ & $\begin{array}{l}\text { média geométrica do total de operações de crédito bancário per } \\
\text { capita nas cidades de origem e de destino. }\end{array}$ & $\begin{array}{l}\text { BANCO } \\
\text { CENTRAL }\end{array}$ \\
\hline EMP & empréstimos & $\begin{array}{l}\mathrm{R} \$ \text { constantes } \\
\text { de jan } / 2015\end{array}$ & $\begin{array}{c}\text { média geométrica do total de operações de empréstimo bancário } \\
\text { (recursos com uso livre) per capita nas cidades de origem e de } \\
\text { destino. }\end{array}$ & $\begin{array}{l}\text { BANCO } \\
\text { CENTRAL }\end{array}$ \\
\hline FIN & financiamentos & $\begin{array}{l}\mathrm{R} \$ \text { constantes } \\
\text { de jan } / 2015\end{array}$ & $\begin{array}{c}\text { média geométrica do total de operações de financiamento } \\
\text { bancário (recursos com uso justificado) per capita nas cidades de } \\
\text { origem e de destino. }\end{array}$ & $\begin{array}{l}\text { BANCO } \\
\text { CENTRAL }\end{array}$ \\
\hline LIQ & liquidez & $\begin{array}{l}\mathrm{R} \$ \text { constantes } \\
\text { de jan } / 2015\end{array}$ & $\begin{array}{c}\text { média geométrica do total de caixa e depósitos bancários per } \\
\text { capita nas cidades de origem e de destino. É uma proxy para o } \\
\text { nível de liquidez vigente nas economias das cidades. }\end{array}$ & $\begin{array}{l}\text { BANCO } \\
\text { CENTRAL }\end{array}$ \\
\hline TREND & tendência & $\mathrm{t}=1,2,3 \ldots \mathrm{T}$ & variável discreta representativa da passagem do tempo. & - \\
\hline ALTA & alta estação & Binária $(0,1)$ & dummy igual a 1 para os meses 1,2 e 12 (verão). & - \\
\hline
\end{tabular}

Na abordagem econométrica adotada, foram realizados testes de hipóteses quanto a problemas típicos da regressão linear e que podem causar problemas de inferência estatística e potencialmente invalidar as conclusões do trabalho, dado que algumas delas representam quebras das hipóteses do modelo econométrico linear clássico (Wooldridge, 2016). Em particular, foram analisados os problemas de endogeneidade, multicolinearidade, heteroscedasticidade, autocorrelação e não-normalidade dos resíduos. Foram realizados testes de hipótese referentes a essas questões, sendo os seus resultados apresentados na Tabela 3.

No que tange à endogeneidade, em qualquer equação de demanda suspeita-se da correlação entre a variável preço e o termo de erro de demanda, o que representa uma quebra de hipóteses do modelo linear clássico, referente à média condicional zero. De fato, é de se esperar que haja vários fatores de demanda não observados pelo analista e que esteja sendo considerados pelas empresas para precificar seu produto. Em diversas situações, assume-se de maneira "ex-ante" a endogeneidade de preços e demanda mesmo sem recorrer a testes de hipótese quanto à endogeneidade, o que é um procedimento ainda mais conservador. Em nosso caso, optamos por 
apresentar o teste na Tabela 3. Trata-se de um teste "C" de diferença de estatísticas de Hansen, do tipo qui-quadrado. Pode-se constatar, pelo resultado do teste, que a variável proxy para preços em nosso modelo, ln YLD, tem sua exogeneidade rejeitada.

Tabela 2 - Estatísticas descritivas das variáveis do modelo empírico

\begin{tabular}{|c|c|c|c|c|c|c|c|c|c|c|c|c|c|}
\hline Variável & PAX & PPC & POP & YLD & LCC & NOV & CSH & CRD & EMP & FIN & LIQ & TREND & ALTA \\
\hline \multicolumn{14}{|l|}{ Correlação } \\
\hline PAX & 1 & & & & & & & & & & & & \\
\hline PPC & 0.343 & 1 & & & & & & & & & & & \\
\hline POP & 0.311 & 0.007 & 1 & & & & & & & & & & \\
\hline YLD & 0.006 & -0.103 & -0.119 & 1 & & & & & & & & & \\
\hline LCC & 0.227 & 0.231 & 0.207 & -0.291 & 1 & & & & & & & & \\
\hline ENT & 0.297 & 0.181 & 0.242 & -0.218 & 0.201 & 1 & & & & & & & \\
\hline $\mathrm{CSH}$ & -0.053 & -0.172 & 0.035 & 0.304 & -0.128 & -0.231 & 1 & & & & & & \\
\hline CRD & 0.579 & 0.803 & 0.109 & -0.129 & 0.244 & 0.270 & -0.210 & 1 & & & & & \\
\hline EMP & 0.581 & 0.703 & 0.162 & -0.155 & 0.269 & 0.269 & -0.281 & 0.937 & 1 & & & & \\
\hline FIN & 0.655 & 0.678 & 0.131 & -0.020 & 0.221 & 0.255 & -0.155 & 0.921 & 1 & 1 & & & \\
\hline LIQ & 0.397 & 0.584 & 0.065 & 0.104 & 0.100 & 0.078 & 0.159 & 0.596 & 0.431 & 0.557 & 1 & & \\
\hline TREND & 0.144 & 0.353 & -0.012 & -0.536 & 0.326 & 0.189 & -0.511 & 0.450 & 0.550 & 0.299 & -0.130 & 1 & \\
\hline ALTA & -0.005 & -0.047 & 0.003 & 0.031 & 0.030 & -0.011 & 0.038 & -0.008 & -0.010 & 0.004 & 0.038 & -0.020 & 1 \\
\hline Variável & PAX & PPC & POP & YLD & LCC & NOV & $\mathrm{CSH}$ & CRD & EMP & FIN & LIQ & TREND & ALTA \\
\hline \multicolumn{14}{|l|}{ Estatísticas } \\
\hline Média & 448.83 & 2081.33 & 254.03 & 0.62 & 0.77 & 0.24 & 0.15 & 1443.65 & 550.09 & 206.32 & 57.01 & 77.20 & 0.25 \\
\hline Desvio Padrão & 874.23 & 763.54 & 242.01 & 0.31 & 0.42 & 0.43 & 0.35 & 1177.68 & 400.28 & 186.54 & 48.32 & 40.94 & 0.43 \\
\hline Mínimo & 30.00 & 489.19 & 2.52 & 0.13 & 0.00 & 0.00 & 0.00 & 36.17 & 23.46 & 1.37 & 4.21 & 1.00 & 0.00 \\
\hline Máximo & 12735.61 & 5510.17 & 1387.96 & 2.84 & 1.00 & 1.00 & 1.00 & 10552.60 & 2614.18 & 1605.93 & 602.60 & 144.00 & 1.00 \\
\hline
\end{tabular}

Foi utilizado um estimador apropriado para o caso de regressor endógeno - o Método dos Momentos Generalizados (MMG). Em sua configuração, são requeridas variáveis adicionais, as chamadas de "variáveis instrumentais", que devem ser variáveis correlacionadas com o regressor endógeno e não correlacionadas com o termo de erro. Utilizou-se uma classe de instrumentos estruturais, ou seja, advindas de um modelo teórico de oferta e demanda para essa função. Foram, assim, utilizadas variáveis de custos médios com insumos de produção das companhias aéreas - combustível, manutenção, seguros e arrendamentos de aeronaves, tarifas aeroportuárias e de navegação aérea, custos das operações em terra, descartando-se custos diretamente relacionados a salários, potencialmente problemáticos por conta de endogeneidade teórica. $\mathrm{Na}$ escolha da combinação ideal de variáveis instrumentais, utilizou-se a abordagem de modelos esparsos de alta dimensão de Belloni, Chen, Chernozhukov e Hansen (2012), que se baseia na escolha ótima de instrumentos por meio de regressão Lasso. Os resultados das regressões finais, entretanto, são de um procedimento MMG pós-Lasso. Os testes de hipótese referentes à qualidade da instrumentação estão reportados no rodapé da tabela final de resultados - vide Tabela 4, Seção 5 -, na forma de testes de instrumentação fraca de Kleibergen-Paap (teste F). Em todas as especificações, pôde-se constatar que os subconjuntos de instrumentos selecionados pela metodologia Lasso passavam nos testes. Experimentamos também com uma especificação em que o número de instrumentos era pequeno, para analisar a robustez do modelo em situação de sobreidentificação mínima. Os resultados não foram modificados nessa especificação, e garantiram também que os instrumentos selecionados passaram no teste de ortogonalidade de Hansen (Teste J), o que confere evidências de que pelo menos um subconjunto dos instrumentos apontados pelo Lasso são potencialmente não-correlacionados com o termo de erro. 
Tabela 3 - Testes de diagnóstico do modelo empírico

\begin{tabular}{|c|c|c|}
\hline Descrição & Estatística & Significância \\
\hline \multicolumn{3}{|l|}{ Teste F de significância conjunta } \\
\hline HO: nulidade de todos os efeitos de rota & 399,53 & $* * *$ \\
\hline \multicolumn{3}{|l|}{ Teste de especificação de Hausman } \\
\hline HO: Diferença entre coeficientes não é sistemática (FE vs RE) & 486,96 & $* * *$ \\
\hline HO: Diferença entre coeficientes não é sistemática (FE vs OLS) & $18.044,76$ & $* * *$ \\
\hline \multicolumn{3}{|l|}{ Teste de endogeneidade } \\
\hline \multicolumn{3}{|l|}{ Teste $\mathrm{C}$ de diferença de estatísticas de Hansen } \\
\hline HO: Regressor exógeno (InYLD) & 97,12 & $* * *$ \\
\hline \multicolumn{3}{|l|}{ Tese de Normalidade para painel de dados } \\
\hline \multicolumn{3}{|l|}{ Teste estendido de Jarque-Bera para o erro variante no tempo } \\
\hline HO: Assimetria da Distribuição Normal & $-0,23$ & \\
\hline HO: Curtose da Distribuição Normal & 0,56 & $* * *$ \\
\hline HO: Teste conjunto de normalidade & 100,77 & $* * *$ \\
\hline \multicolumn{3}{|l|}{ Teste de Heteroscedasticidade } \\
\hline \multicolumn{3}{|l|}{ HO: independência entre cortes transversais } \\
\hline \multicolumn{3}{|l|}{ Teste geral de Pagan-Hall } \\
\hline usando níveis dos regressores apenas & $7.007,38$ & $* * *$ \\
\hline usando níveis e quadrados dos regressores & $7.138,01$ & $* * *$ \\
\hline \multicolumn{3}{|l|}{ Teste de Autocorrelação } \\
\hline \multicolumn{3}{|l|}{ H0: resíduos serialmente não correlacionados } \\
\hline Teste de Cumby-Huizinga (ordem 1) & $6.164,04$ & $* * *$ \\
\hline Teste de Cumby-Huizinga (ordem 3) & $6.664,41$ & $* * *$ \\
\hline Teste de Cumby-Huizinga (ordem 12) & $7.435,68$ & $* * *$ \\
\hline Teste de Cumby-Huizinga (ordem 24) & $7.567,55$ & $* * *$ \\
\hline \multicolumn{3}{|l|}{ Teste de Multicolinearidade } \\
\hline \multicolumn{3}{|l|}{ HO: Ausência de multicolinearidade } \\
\hline Farrar-Glauber Qui2-Test & $216.694,55$ & $* * *$ \\
\hline \multicolumn{3}{|l|}{ Variance Inflation Factor (VIF) } \\
\hline Estatística VIF média & 398,78 & \\
\hline Estatística VIF máxima & $3.474,47$ & \\
\hline
\end{tabular}

Notas: representação dos valores-p dos testes (significância): *** $\mathrm{p}<0.01$;

Com relação à multicolinearidade, tem-se que a situação mais comum é gerada por potenciais correlações altas entre regressores levando a uma falta de significância estatística artificial, incrementando o risco de falso negativo nos testes de hipóteses individuais das variáveis. Para se avaliar as associações entre regressores, costuma-se analisar a matriz de correlações de Pearson, disponível na Tabela 2. Pode-se notar que, naquela tabela, algumas das correlações entre os regressores são altas (por exemplo, referentes às variáveis de crédito e a de renda per capita), o que é sugestivo de vigência de alta multicolinearidade. Adicionalmente às análises das correlações, para o estudo de multicolinearidade, pode-se rodar um teste do tipo qui-quadrado de Farrar-Glauber e também extrair a estatística VIF (Variance Inflation Factor). Os resultados do teste e estatísticas estão apresentados na Tabela 3 e são bastante confirmatórios da problemática. Em vigência de multicolinearidade, deve-se interpretar com cautela os resultados de rejeição de significância de regressores.

O modelo econométrico linear clássico assume que os erros da regressão apresentam variância constante (homoscedasticidade). No entanto, essa premissa é dificilmente satisfeita em 
casos práticos, e, portanto, temos que as bases de dados em geral disponíveis apresentam estimativas de resíduos tipicamente heteroscedásticos. De maneira análoga, em se tratando de painel de dados com um conjunto grande de períodos próximos entre si (no caso, 144, com periodicidade mensal), também é de se esperar que os resíduos sejam serialmente correlacionados, ou seja, que vigore alguma forma de autocorrelação dos mesmos. Em ambos os casos (heteroscedasticidade e autocorrelação dos resíduos), temos uma situação de maior risco de falso positivo nos testes de hipóteses isolados das variáveis do modelo de regressão (Wooldridge, 2016). Com o objetivo de analisar a existência desses potenciais problemas na base de dados utilizada nas regressões, foram aplicados os testes de Cumby-Huizinga (autocorrelação) e de Pagan-Hall (heteroscedasticidade). Os resultados estão apresentados na Tabela 3, e rejeitam as respectivas hipóteses nulas. Com o intuito de mitigar os efeitos da existência de autocorrelação e de heteroscedasticidade na base de dados utilizada, utilizou-se o procedimento corretivo de selecionar estimadores consistentes com a presença dessas anomalias presentes nos dados. Dessa maneira, o método utilizado para estimar o modelo empírico foi o de estimação por efeitos fixos implementado pelo MMG com erros-padrões robustos e eficientes para autocorrelação e para heteroscedasticidade (o chamado estimador de Newey-West, conforme em Wooldridge, 2016, p. 390). Para o cômputo das estimativas, foi utilizado o software Stata 14.1.

Por fim, foi testada a questão da não-normalidade dos resíduos da regressão, com uso do teste estendido para painéis de dados de Jarque-Bera. Os resultados dos testes estão apresentados na Tabela 3. As estatísticas quanto à assimetria e curtose da distribuição dos resíduos mostram que, enquanto a primeira propriedade é satisfeita, há evidências de rejeição da segunda propriedade na amostra. Por outro lado, Wooldrige (2016, p. 107 e 124), argumenta que a não-normalidade dos erros não é um problema sério para tamanhos de amostra grandes, situação do presente caso (45.585 observações). 0 autor ainda discorre que (pp. 150 e 155) a normalidade não desempenha papel na propriedade de ausência de viés do estimador de mínimos quadrados e que, adicionalmente, para amostras grandes, as estatísticas calculadas "t" dos modelos se distribuem aproximadamente como a distribuição t-Student mesmo em situações em que a premissa de normalidade dos resíduos não seja válida. Sendo assim, interpretamos os testes de hipótese quanto à normalidade dos resíduos como um procedimento adicional de conhecimento do processo gerador dos dados e, dado o tamanho da amostra, adotamos o procedimento de não invalidar os testes de hipóteses obtidos por conta da propriedade do estimador continuar sendo o melhor estimador linear não-enviesado - i.e., continua atendendo as premissas do Teorema de Gauss-Markov - nesses casos.

\subsection{Validação Cruzada}

Com o intuito de promover uma discussão acerca do comportamento fora da amostra da modelagem empírica proposta, foi utilizado o método da Validação Cruzada, também chamada de "estimação rotacional" ou procedimento sistemático de testes fora da amostra (out-of-sample testing). A Validação Cruzada é uma técnica estatística usada para verificar a capacidade de generalização de um modelo a partir de um conjunto de dados (Kohavi, 1995). 0 principal objetivo da Validação Cruzada é testar a capacidade de previsão do modelo, o que permite identificar problemas estatísticos como o "sobreajuste" (overfiting) - quando o modelo se ajusta bem aos dados, mas é ineficaz em previsões de novas realizações da amostra (Cawley e Talbot, 2010).

Existem diversos tipos de algoritmos de Validação Cruzada, como os métodos "holdout", "kfold" e "leave-one-out", sendo que todos eles são baseados no princípio de se dividir uma base 
de dados em diversos subconjuntos, em que um subconjunto é utilizado para a estimação do modelo sob análise, enquanto os outros subconjuntos são utilizados para a validação do mesmo por meio de previsões fora da amostra. No cerne do procedimento de Validação Cruzada está a premissa de que um bom modelo deveria não apenas permitir um bom ajuste a uma dada amostra, mas também permitir prever o comportamento de informações não amostrais.

0 método de Validação Cruzada utilizado neste trabalho foi o $k$-fold. Esse método divide aleatoriamente a base de dados em $k$-subconjuntos de mesmo tamanho, e então um subconjunto é utilizado para o teste do modelo enquanto os outros $k$ - 1 subconjuntos restantes são utilizados para a validação do primeiro, calculando-se a qualidade das previsões do modelo. Este processo é repetido $k$ vezes, com cada subconjunto utilizado apenas uma vez para o teste do modelo. Ao final das $k$ iterações, analisa-se a qualidade da previsão fora da amostra do modelo, a partir das $k$ estimativas de erros de previsão encontrados - em geral extrai-se o erro quadrático médio das previsões fora da amostra -, obtendo, assim, uma medida da capacidade do modelo de representar o processo gerador de dados (Kohavi, 1995). Uma das vantagens dessa abordagem em relação às demais é que este é um método não exaustivo, ou seja, não testa todas as maneiras possíveis de dividir o conjunto de dados em subconjuntos de estimação e validação, permitindo uma rápida e eficiente análise do modelo. Além disso, dos métodos não exaustivos, o método $k$ fold tem a propriedade de garantir que todos os subconjuntos sejam utilizados tanto para o teste do modelo quanto para a validação, e que todos os subconjuntos gerados sejam utilizados ao menos uma vez para o teste do modelo. 0 método foi configurado com o número de subdivisões iguais a 5 e 10, sendo extraídas as estatísticas de erro quadrático médio da previsão fora da amostra para cada estimação. Na tabela final de resultados, apresentada na Seção 5 (Tabela 4) são reportados, abaixo de cada estimação, os valores máximos de erro de previsão estimados, denominados de "RMSE CV-K5 (Máx)" e "RMSE CV-K10 (Máx)”. Em todos os casos, os valores de RMSE foram suficientemente baixos e similares aos valores de RMSE do modelo de regressão, sugerindo que os modelos propostos não sofrem de problemas de previsão fora da amostra e de sobreajuste.

\section{RESULTADOS}

Os resultados obtidos com a estimação dos parâmetros do modelo empírico podem ser observados na Tabela 4. A Coluna (1) apresenta o modelo apenas com as variáveis causais (ou seja, as com resultado esperado ex-ante, ln PPC, ln POP e ln YLD) e algumas variáveis dummy de controle (LCC, ENT e CSH), sem nenhuma variável referente ao crédito. Na Coluna (2), insere-se a variável ln CRD (total de crédito per capita). Na Coluna (3), insere-se, em substituição à variável agregada de total de crédito, insere-se uma quebra entre empréstimos e financiamentos per capita - respectivamente, as variáveis ln EMP e ln FIN. A especificação da Coluna (4) apresenta os resultados com a inclusão da proxy para liquidez da economia (ln LIQ), especificação a qual consideramos a principal. Já as especificações das Colunas (5), (6) e (7) apresentam resultados de checagem de robustez dos resultados principais: em (5), utiliza-se apenas duas variáveis instrumentais na identificação (modelo com sobreidentificação mínima), em (6) é descartada o controle LCC e em (7) são incluídos controles de tendência e sazonalidade - respectivamente, TREND e ALTA.

Além de apresentarem informações relevantes acerca da influência do crédito na demanda por transporte aéreo, os resultados das distintas modelagens também ajudam a averiguar a robustez da estratégia empírica. Considerando que os coeficientes estimados de ln PPC, ln POP e 
ln YLD não apresentam trocas de sinais e de significância estatística ao longo das colunas, podese inferir que o modelo apresenta robustez em suas variáveis causais. Já os controles indicativos de presença de empresas originadas do modelo low cost (LCC) e empresas novatas menores (EMP), foi obtido o resultado que essas empresas estão associadas a uma geração maior de demanda, muito provavelmente por conta da maior concorrência em frequência de voos e das campanhas de publicidade que acompanham a sua entrada no mercado. É importante notar que o coeficiente estimado da variável LCC é pelo menos seis vezes maior que o coeficiente estimado da variável ENT, sendo indicativo que o poder de geração de demanda nova das primeiras é bastante superior. A variável CSH apresentou coeficiente positivo e estatisticamente significante, resultado esse que não se manteve nas especificações de checagem de robustez das Colunas de (5) a (7).

Tabela 4 - Resultados da estimação do modelo empírico

\begin{tabular}{|c|c|c|c|c|c|c|c|}
\hline & $\begin{array}{l}\text { (1) } \\
\text { In PAX }\end{array}$ & $\begin{array}{l}\text { (2) } \\
\text { In PAX }\end{array}$ & $\begin{array}{l}\text { (3) } \\
\text { In PAX }\end{array}$ & $\begin{array}{l}\text { (4) } \\
\text { In PAX }\end{array}$ & $\begin{array}{l}\text { (5) } \\
\text { In PAX }\end{array}$ & $\begin{array}{l}\text { (6) } \\
\text { In PAX }\end{array}$ & $\begin{array}{l}\text { (7) } \\
\text { In PAX }\end{array}$ \\
\hline In PPC & $\begin{array}{l}0.4568^{* * *} \\
{[0.049]}\end{array}$ & $\begin{array}{l}0.2469 * * * \\
{[0.044]}\end{array}$ & $\begin{array}{l}0.1700^{* * *} \\
{[0.041]}\end{array}$ & $\begin{array}{l}0.1778^{* * *} \\
{[0.041]}\end{array}$ & $\begin{array}{l}0.3270^{* * *} \\
{[0.041]}\end{array}$ & $\begin{array}{l}0.2046^{* * *} \\
{[0.044]}\end{array}$ & $\begin{array}{l}0.2533^{* * *} \\
{[0.040]}\end{array}$ \\
\hline In POP & $\begin{array}{l}1.5697^{* * *} \\
{[0.100]}\end{array}$ & $\begin{array}{l}1.0159^{* * *} \\
{[0.104]}\end{array}$ & $\begin{array}{l}0.7418^{* * *} \\
{[0.117]}\end{array}$ & $\begin{array}{l}0.9359^{* * *} \\
{[0.120]}\end{array}$ & $\begin{array}{l}0.3991^{* * *} \\
{[0.125]}\end{array}$ & $\begin{array}{l}1.6411^{* * *} \\
{[0.125]}\end{array}$ & $\begin{array}{l}0.4365^{* * *} \\
{[0.152]}\end{array}$ \\
\hline In YLD & $\begin{array}{l}-0.8274 * * * \\
{[0.034]}\end{array}$ & $\begin{array}{l}-0.7208^{* * *} \\
{[0.037]}\end{array}$ & $\begin{array}{l}-0.6602 * * * \\
{[0.044]}\end{array}$ & $\begin{array}{l}-0.8394 * * * \\
{[0.046]}\end{array}$ & $\begin{array}{l}-0.3295 * * * \\
{[0.067]}\end{array}$ & $\begin{array}{l}-0.7065^{* * *} \\
{[0.048]}\end{array}$ & $\begin{array}{l}-0.6748^{* * *} \\
{[0.039]}\end{array}$ \\
\hline LCC & $\begin{array}{l}0.2837^{* * *} \\
{[0.009]}\end{array}$ & $\begin{array}{l}0.2815^{* * *} \\
{[0.009]}\end{array}$ & $\begin{array}{l}0.2801^{* * *} \\
{[0.009]}\end{array}$ & $\begin{array}{l}0.2909 * * * \\
{[0.009]}\end{array}$ & $\begin{array}{l}0.2892^{* * *} \\
{[0.008]}\end{array}$ & & $\begin{array}{l}0.2808^{* * *} \\
{[0.009]}\end{array}$ \\
\hline ENT & $\begin{array}{l}0.0291^{* * *} \\
{[0.008]}\end{array}$ & $\begin{array}{l}0.0438^{* * *} \\
{[0.008]}\end{array}$ & $\begin{array}{l}0.0420 * * * \\
{[0.008]}\end{array}$ & $\begin{array}{l}0.0404 * * * \\
{[0.008]}\end{array}$ & $\begin{array}{l}0.0739 * * * \\
{[0.008]}\end{array}$ & $\begin{array}{l}0.0283^{* * *} \\
{[0.008]}\end{array}$ & $\begin{array}{l}0.0505^{* * *} \\
{[0.007]}\end{array}$ \\
\hline $\mathrm{CSH}$ & $\begin{array}{l}0.0450^{* * *} \\
{[0.009]}\end{array}$ & $\begin{array}{l}0.0426^{* * *} \\
{[0.009]}\end{array}$ & $\begin{array}{l}0.0522 * * * \\
{[0.009]}\end{array}$ & $\begin{array}{l}0.0359 * * * \\
{[0.009]}\end{array}$ & $\begin{array}{l}0.0130 \\
{[0.009]}\end{array}$ & $\begin{array}{l}0.0339 * * * \\
{[0.009]}\end{array}$ & $\begin{array}{l}0.0130 \\
{[0.009]}\end{array}$ \\
\hline In CRD & & $\begin{array}{l}0.1980^{* * *} \\
{[0.019]}\end{array}$ & & & & & \\
\hline In EMP & & & $\begin{array}{l}0.2467^{* * *} \\
{[0.026]}\end{array}$ & $\begin{array}{l}0.1476^{* * *} \\
{[0.027]}\end{array}$ & $\begin{array}{l}0.3887^{* * *} \\
{[0.035]}\end{array}$ & $\begin{array}{l}0.2103^{* * *} \\
{[0.028]}\end{array}$ & $\begin{array}{l}0.0966^{* * *} \\
{[0.021]}\end{array}$ \\
\hline In FIN & & & $\begin{array}{l}0.0258^{* *} \\
{[0.013]}\end{array}$ & $\begin{array}{l}0.0024 \\
{[0.013]}\end{array}$ & $\begin{array}{l}0.0329 * * \\
{[0.013]}\end{array}$ & $\begin{array}{l}0.0304^{* *} \\
{[0.013]}\end{array}$ & $\begin{array}{l}-0.0026 \\
{[0.013]}\end{array}$ \\
\hline In LIQ & & & & $\begin{array}{l}0.0717^{* * *} \\
{[0.008]}\end{array}$ & $\begin{array}{l}0.0467 * * * \\
{[0.008]}\end{array}$ & $\begin{array}{l}0.0394 * * * \\
{[0.008]}\end{array}$ & $\begin{array}{l}0.0684^{* * *} \\
{[0.007]}\end{array}$ \\
\hline TREND & & & & & & & $\begin{array}{l}0.0019 * * * \\
{[0.000]}\end{array}$ \\
\hline ALTA & & & & & & & $\begin{array}{l}0.0536^{* * *} \\
{[0.006]}\end{array}$ \\
\hline R2 Ajustado & 0.9073 & 0.9119 & 0.9139 & 0.9130 & 0.9200 & 0.9075 & 0.9167 \\
\hline RMSE & 0.3566 & 0.3476 & 0.3436 & 0.3454 & 0.3317 & 0.3561 & 0.3385 \\
\hline RMSE CV-K5 (Máx) & 0.3526 & 0.3449 & 0.3420 & 0.3411 & 0.3412 & 0.3542 & 0.3367 \\
\hline RMSE CV-K10 (Máx) & 0.3562 & 0.3497 & 0.3539 & 0.3512 & 0.3432 & 0.3570 & 0.3382 \\
\hline N. Var. Instrumentais & 9 & 10 & 10 & 9 & 2 & 9 & 8 \\
\hline F (Identif. Fraca) & 210.06 & 155.74 & 118.04 & 128.90 & 189.06 & 128.62 & 188.06 \\
\hline N. Observações & 45.585 & 45.585 & 45.585 & 45.585 & 45.585 & 45.585 & 45.585 \\
\hline
\end{tabular}

Os resultados das Colunas (2) a (4) mostram evidências de que o comportamento do consumidor do transporte aéreo é potencialmente afetado pelas condições de crédito na economia local. De fato, a variável ln CRD (Coluna 2) possui coeficiente estimado positivo e estatisticamente significante. Nas Colunas (3) e (4), observa-se que esse resultado é mais evidente para os empréstimos bancários (variável ln EMP), que também possui coeficiente estimado positivo e estatisticamente significante em ambas as colunas. Já a variável referente aos financiamentos 
bancários (ln FIN) foi estatisticamente significante apenas na especificação da Coluna (3), perdendo sua significância na Coluna (4), quando a variável ln LIQ foi inserida. Isso é indicativo que o poder explicativo dessa variável na demanda por transporte aéreo é correlacionado ao estado da liquidez da economia e que provavelmente é não existente ("confusão" de efeitos). De fato, não é prática comum no segmento bancário o parcelamento de passagens aéreas com uso de financiamento, evidência que é consistente com os resultados.

Com relação à checagem de robustez dos resultados do modelo da Coluna (4), ou seja, os demais resultados, contidos nas Colunas (5), (6) e (7), pode-se concluir que, à exceção das variáveis CSH e ln FIN, todas as demais variáveis se mantiveram estatisticamente significantes e com seus respectivos sinais mantidos. Em particular, em referência às variáveis de interesse para o presente estudo - nossas variáveis de experimentação -, respectivamente, ln CRD, ln EMP e ln FIN, é possível observar que as duas primeiras se mantêm com resultados inalterados ao longo das colunas, mesmo ao se introduzirem desafios metodológicos adicionais à especificação principal em (4). Assim, verifica-se robustez dos resultados nos casos de sobreidentificação mínima (Coluna 5), subespecificação do efeito LCC (Coluna 6), e controles de tendência e sazonalidade (Coluna 7). Importante salientar, que todas as especificações passaram por um procedimento prévio de regressão Lasso, onde não apenas as variáveis instrumentais, como também os regressores, estavam passíveis de serem descartados do modelo, o que não aconteceu com os regressores da Tabela 4.

\section{CONSIDERAÇÕES FINAIS}

O presente trabalho desenvolveu uma modelagem econométrica para analisar e testar empiricamente o efeito da disponibilidade de crédito bancário sobre a demanda por transporte aéreo no país. A partir da estimação da relação entre variáveis indicativas de crédito per capita nas economias locais de origem e destino, e a demanda por viagens aéreas, foi possível compreender de uma maneira agregada os efeitos potencialmente exercidos sobre famílias e empresas, enquanto consumidores do transporte aéreo, e advindos da maior disponibilidade de diversos tipos de mecanismos financeiros e bancários no país. Assim, foi possível explicitamente testar e encontrar evidências acerca da hipótese de existência de impacto efetivo do crédito sobre o movimento de passageiros nas rotas domésticas brasileiras.

Considerado os resultados obtidos no modelo, foi possível observar que as variáveis relacionadas à presença de crédito na economia apresentaram resultados estatisticamente significantes em diversas especificações, sugerindo que alguma proxy para as condições de renda/riqueza na forma de acesso ao crédito deve ser considerada em exercícios de modelagens de demanda por transporte aéreo de países emergentes. Os resultados sugerem ainda que a maior disponibilidade de crédito na economia fornece condições para que novos consumidores experimentem o transporte aéreo, aumentando a acessibilidade econômica ao seu consumo e incrementando as taxas de crescimento do setor. As relações estimadas, entretanto, possuem a limitação de se tratarem de efeitos de curto e médio prazo apenas, não podendo ser extrapoladas em análises de longo prazo que incorporem os efeitos perversos da dinâmica de endividamento das famílias. Adicionalmente, o estudo não englobou o período mais recente, de recessão, sendo, portanto, restrito a um período de maior crescimento econômico apenas.

\section{AGRADECIMENTOS}

Coordenação de Aperfeiçoamento de Pessoal de Nível Superior (Capes) - Programa de Demanda Social (DS), Fundação de Amparo à Pesquisa do Estado de São Paulo (FAPESP) - Auxílio Pesquisa n. 2015/19444-1; Conselho Nacional de Desenvolvimento 
Científico e Tecnológico (CNPq), Auxílio n. 301344/2017-5. Os autores gostariam de agradecer os comentários de Frederico A. Turolla, Giovanna Ronzani, Daniel Pamplona, do editor e dos pareceristas anônimos. Todos os eventuais erros e omissões são de nossa autoria.

\section{REFERÊNCIAS}

ANAC. (2016) 10 anos - Agência Nacional de Aviação Civil. 1st. ed. Brasília: ANAC, 195 p.

Bacchetta, P. e Gerlach, S. (1997). Consumption and credit constraints: International evidence. Journal of Monetary Economics, v. 40, n. 2, p. 207-238. DOI: 10.1016/S0304-3932(97)00042-1.

Belloni, A., Chen, D., Chernozhukov, V. e Hansen, C. (2012). Sparse models and methods for optimal instruments with an application to eminent domain. Econometrica, v. 80, n. 6, p. 2369-2429. DOI: 10.3982/ECTA9626.

Boonekamp, T., Zuidberg, J. e Burghouwt, G. (2018). Determinants of air travel demand: The role of low-cost carriers, ethnic links and aviation-dependent employment. Transportation Research Part A: Policy and Practice, v. 112, p. 18-28. DOI: 10.1016/j.tra.2018.01.004.

Borça Jr., G. e Guimarães, D. (2015). Impacto do ciclo expansionista de crédito à pessoa física no desempenho da economia brasileira 2004-2013. Revista do BNDES, n. 43, p. 157-159.

Jorge-Calderón, J. D. (1997). A demand model for scheduled airline services on international European routes. Journal of Air Transport Management, v. 3, n. 1, p. 23-35. DOI: 10.1016/S0969-6997(97)82789-5.

Cawley, G. C. e Talbot, N. L. (2010). On over-fitting in model selection and subsequent selection bias in performance evaluation. Journal of Machine Learning Research, v. 11, p. 2079-2107.

Di Maggio, M. e Kermani, A. (2017). Credit-induced boom and bust. The Review of Financial Studies, v. 30, n. 11, p. $3711-3758$. DOI: $10.1093 / \mathrm{rfs} / \mathrm{hhx} 056$.

Galeano, E. V. e Feijó, C. (2012). Crédito e crescimento econômico: evidências a partir de um painel de dados regionais para a economia brasileira nos anos 2000. Revista Econômica do Nordeste, v. 43, n. 2, p. 201-220.

Gall, N. (2008) A festa do crédito e a economia mundial: Dinheiro, ganância, tecnologia. Braudel Papers, n. 43.

Grosche, T., Rothlauf, F. e Heinzl, A. (2007). Gravity models for airline passenger volume estimation. Journal of Air Transport Management, v. 13, n. 4, p. 175-183. DOI: 10.1016/j.jairtraman.2007.02.001.

Kazda, A. e Caves, R. (2015) Airport Design and Operation. 3a. ed. Bingley: Emerald Group Publishing Limited. 569p.

Kohavi, R. (1995). A study of cross-validation and bootstrap for accuracy estimation and model selection. In International Joint Conference on Artificial Intelligence, v. 2, p. 1137-1143.

Maddala, G. S. e Lahiri, K. (1992). Introduction to econometrics, v. 2. New York: Macmillan.

Olney, M. L. (1999). Avoiding default: The role of credit in the consumption collapse of 1930. The Quarterly Journal of Economics, v. 114, n. 1, p. 319-335. DOI: 10.1162/003355399555927.

Sant'Anna, A. A., Borça Jr., G. e Araujo, P. Q. (2009). Mercado de crédito no Brasil: evolução recente e o papel do BNDES (20042008). Revista do BNDES, v. 16, n. 31, p. 58-59.

Soman, D. e Cheema, A. (2002) The effect of credit on spending decisions: The role of the credit limit and credibility. Marketing Science, v. 21, n. 1, p. 32-53. DOI: 10.1287/mksc.21.1.32.155.

Valdes, V. (2015). Determinants of air travel demand in Middle Income Countries. Journal of Air Transport Management, v. 42 , p. 75-84. DOI: 10.1016/j.jairtraman.2014.09.002.

Wooldridge, J. M. (2016). Introductory econometrics: A modern approach. Nelson Education. 